FACTA UNIVERSITATIS

Series: Mechanical Engineering Vol. 18, No 1, 2020, pp. 121 - 134

https://doi.org/10.22190/FUME191129013T

Original scientific paper

\title{
THE SELECTION OF OPTIMAL REVERSIBLE TWO-SPEED PLANETARY GEAR TRAINS FOR MACHINE TOOL GEARBOXES
}

\author{
Sanjin Troha ${ }^{1}$,̌eljkoVrcan ${ }^{1}$, Dimitar Karaivanov ${ }^{2}$, \\ Madina Isametova ${ }^{3}$ \\ ${ }^{1}$ University of Rijeka, Faculty of Engineering, Croatia \\ ${ }^{2}$ University of Chemical Technology and Metallurgy, Sofia, Bulgaria \\ ${ }^{3}$ Satbayev University, Almaty, Kazakhstan
}

\begin{abstract}
The application of multi-criteria optimization to two-carrier two-speed planetary gear trains is outlined in this paper. In order to determine the mathematical model of multi-criteria optimization variables, the objective functions and conditions must be determined first. Two-carrier two-speed planetary gear trains with brakes on coupled shafts are analyzed in this paper. The mathematical model covers the determination of the set of the Pareto optimal solutions as well as the method for selecting an optimal solution from this set. A numerical example is provided to illustrate the procedure in which the optimal two-speed planetary gear train is selected and defined by design parameters.
\end{abstract}

Key Words: Multi-criteria Optimization, Two-speed Planetary Gear Trains, Pareto Optimal Solutions, Coupled Shafts

\section{INTRODUCTION}

Multi-criteria optimization problems are very common in many scientific and technical solutions. The optimization of gear trains as complete technical systems implies a complex mathematical model that has to describe actual system operation in actual circumstances. Planetary gear trains (PGT)s are a type of geared transmission which offers many advantages in comparison to conventional gearboxes. Therefore, the area of application of single-stage and multi-stage PGTs in mechanical engineering is increasing. Multi-stage PGTs are obtained by linking the shafts of one or two single stage PGTs. A special multi-stage PGT is a two-speed, two-carrier PGT consisting of two coupling shafts and four external shafts. This type of compound gear train has many important characteristics, the most notable being the ability to change the transmission ratio and the direction of rotation of the output

Received November 29, 2019 / Accepted March 03, 2020

Corresponding author: Sanjin Troha

University of Rijeka, Faculty of Engineering, Vukovarska ul. 58, 51000, Rijeka, Croatia

E-mail: sanjin.troha@riteh.hr 
shaft under load on demand. Therefore, they are particularly suited for applications as main drives in machinery, e.g. machine tools, cranes, etc.

\subsection{State of the art}

The application of multi-criteria optimization to gear transmissions, especially planetary gear transmissions has not been the subject of many research papers. A population-based evolutionary multi-objective optimization approach, based on the concept of Pareto optimality, is proposed in paper [1] for the design of helical gears. Paper [2] deals with the selection of the best parameters in order to obtain the required gear quality and with the optimization of the design process itself. An analytical and computer aided procedure for the multi-criteria design optimization of multi-stage gear transmission is presented in paper [3]. The process of planetary gear transmission optimization is shown in paper [4] as a method which leads to the optimal solution.On the other hand, there were very few research efforts dealing with two-speed, two-carrier PGTs until 2003 [5]. Two-carrier PGTs consisting of two coupled and four external shafts, which enable two-speed transmissions, have significant application as gearboxes [5]. The possible schemes of these transmissions are presented in [5-10], while possible transmission structures with convenient brake layouts which could be used as two-speed transmissions are examined in [5]. A method for investigating the transmission ratio, the internal power flows and the efficiency of complex multi-carrier gearings is presented in [7]. An optimization of the two-carrier two-speed PGTs with brakes on single shafts is provided in [11]. In this example, a fishing boat transmission was chosen as input data for the numerical example of multi-criteria gearbox optimization.

This paper provides an optimization of the two-carrier two-speed PGTs with brakes on the coupled shafts, in continuation of the optimal selection choice methodology application. The characteristics of a machine tool transmission have been used as input data for the numerical example of multi-criteria optimization application. Apart from the determination of the set of the Pareto optimal solutions, the weighted coefficient method was applied in order to determine the optimal solution.

\section{Mathematical Model for Planetary Gear Train Optimization}

The two-carrier two-speed PGTs with brakes on coupled shafts are built from basic types of PGT. The basic type of PGT (type 2k-h, variant A) is an arrangement using a central sun gear with external gearing (1), external ring gear with internal gearing (3), planet gears with external gearing(2) and planet carrier (h), as shown in Fig.1. The planets are in simultaneous mesh with the sun gear and the ring gear. Also, a WolfArnaudov's symbol can be used, indicating the torque on the main elements as a function of basic transmission ratio $i_{0}$. The equations for the basic transmission ratio and the ideal torque ratio calculation are also pointed out in Fig. 1. The carrier shaft is the summary element of the basic PGT, as a negative transmission ratio is obtained by stopping the planet carrier, indicating a change of the direction of rotation of the output element. 


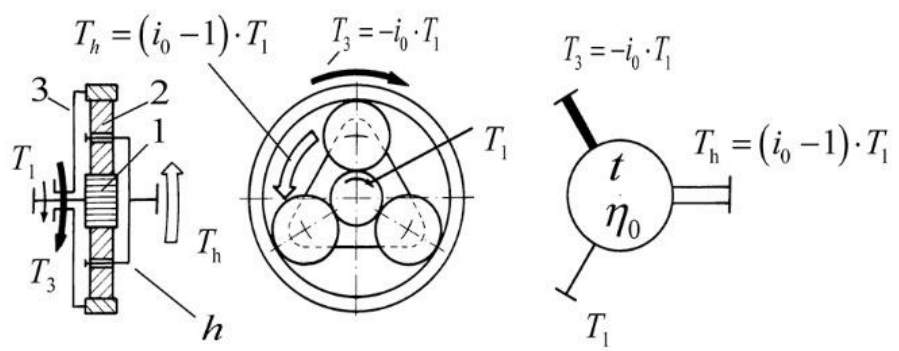

Basic transmission ratio: $i_{0}=z_{3} / z_{1}<0$. Ideal torque ratio: $t=\frac{T_{3}}{T_{1}}=\frac{\left|z_{3}\right|}{z_{1}}=-i_{0}>+1$.

Fig. 1 Basic type of PGT and Wolf-Arnaudov's symbol with torque ratios (1 - sun gear; 2 - planet; 3 - ring gear; $\mathrm{h}$ - planet carrier)

The process of finding the optimal solution starts with the definition of a mathematical model, as stated in [12]. The complete mathematical model of the basic type of PGT was described in the aforementioned paper and a brief summary will be also presented in this section.

It is necessary to define variables, objective functions and functional constraints in order to define a mathematical model.

\subsection{Variables}

The following variables are considered by this model: the number of teeth of sun gear $z_{1}$, the number of teeth of planet gears $z_{2}$, the number of teeth of ring gear $z_{3}$, the number of planets $n_{w}$, gear module $m_{n}$ and gear face width $b$.

The optimization variables are of the mixed type: the gear tooth numbers are positive and negative integers, the number of planets is a discrete value, the module is a discrete standard value (acc. to ISO 54), while the face width is a continuous variable. The gear tooth numbers and the number of planets are non-dimensional values while the module and the face width are given in millimeters.

\subsection{Objective functions}

The characteristics used by the model to determine the objective functions are the volume, mass, efficiency and manufacturing cost of PGTs.

The volume of the PGTs is used as an overall dimension expression, and the gears are approximated with a cylinder volume with the diameter equal to the pitch diameter and the height equal to the face width. The fact that the planets are inside the ring gear makes it possible for the PGT volume to be expressed by Eq. (1)

$$
V=\frac{\pi}{4} \cdot b \cdot\left(\frac{m_{n} \cdot z_{3}}{\cos \beta} \cdot \frac{\cos \alpha_{t}}{\cos \alpha_{w t 23}}\right)^{2}
$$

where $\alpha_{t}$ is the transverse pressure angle, $\alpha_{w t 23}$ is the working transverse pressure angle for the pair 2-3 and $\beta$ is the helix angle at the pitch diameter. 
Since the mass of a particular gear is determined as gear volume multiplied by the density of gear material and fact that mass is determined as the sum of all gear masses in a PGT, this criterion has been expressed as Eq. (2):

$$
m=0.25 \cdot \pi \cdot b \cdot \rho \cdot \frac{m_{n}^{2}}{\cos ^{2} \beta} \cdot\left[k_{1} \cdot z_{1}^{2} \cdot \frac{\cos ^{2} \alpha_{t}}{\cos ^{2} \alpha_{w t 12}}+n_{w} \cdot k_{2} \cdot z_{2}^{2} \cdot \frac{\cos ^{2} \alpha_{t}}{\cos ^{2} \alpha_{w t 12}}+k_{3} \cdot z_{3}^{2} \cdot \frac{\cos ^{2} \alpha_{t}}{\cos ^{2} \alpha_{w t 23}}\right]
$$

Efficiency is one of the most important criteria for the design and evaluation of the arrangement quality. The calculation of the gear transmission efficiency is generally confined to losses depending on the friction on tooth flanks in contact while neglecting the losses in bearings and losses due to oil viscosity, i.e. restricted to the calculation of contact power losses [12-14]. The model, followed by the developed computer program, is adjusted to the most commonly used variant with the sun gear as the input element, and the carrier as the output element while the ring gear remains stationary. Basic PGT efficiency in this case is given by Eq. (3) [12]:

$$
\eta=\frac{1-i_{0} \cdot \eta_{0}}{1-i_{0}}
$$

where $\eta_{0}$ is the efficiency with the planet carrier stationary, as expressed by Eq. (4)

$$
\eta_{0}=1-\frac{z_{3}}{z_{3}-z_{1}}\left(\frac{0.15}{z_{1}}+\frac{0.35}{z_{2}}+\frac{0.20}{z_{3}}\right)
$$

The economic demands must be also taken into consideration in the technoeconomical optimization, as these demands are directly related to production costs. The time needed for the manufacture of gears is taken as a measure of the production costs and as an economic factor. This function is then determined as a sum of the time periods needed for the manufacturing of sun gear $\left(T_{P 1}\right)$, planets $\left(T_{P 2}\right)$ and ring gear $\left(T_{P 3}\right)$, i.e.

$$
F_{T}=T_{1}+n_{w} \cdot T_{2}+T_{3}
$$

The production times are determined according to Fette, Lorenc and Höfler [15].

\subsection{Functional constraints}

The functional constraints are the conditions required for the proper operation of a system. There are numerous exceptions that need to be taken into consideration for PGTs to operate correctly in comparison to conventional gear transmissions. The exceptions presented in this model are related to assembly conditions, geometrical conditions and strength conditions. The assembly conditions include the conditions of coaxiality, adjacency and conjunction [16].

The geometrical conditions are related to the undercutting and profile interference, the ratio of the pressure angle to the working transverse pressure angle, the tooth thickness and the tooth space width, the transverse contact ratio value, the sliding speeds at the point of contact, the ratio of the pinion face width to the pinion reference diameter, etc. These conditions have been ensured in accordance with the actual standards (ISO TC 60 list of Standards 090915).

The strength conditions, safety factors for bending strength and surface durability of each gear, are checked according to ISO 6336-1 to ISO 6336-3 [17]. 


\subsection{Steps in the optimization process}

The optimization process begins by generating all solutions for the assigned input data. All 6-tuples of design parameters $\left(z_{1}, z_{2}, z_{3}, n_{w}, m_{n}, b\right)$ satisfying the functional constraints are generated for the given input data (transmission ratio, input number of revolution, input torque, service life in hours, application factor, accuracy grade Q(DIN 3961), and the values of the objective functions for every 6-tuple are computed. These 6tuples form a set of feasible solutions. An optimal solution is then selected, based on the established objective functions and constraints, and determined by variables.

The mathematical model of nonlinear multi-criteria problem can be formulated as follows:

$$
\begin{aligned}
& \max \left\{f_{1}(x), f_{2}(x), \ldots, f_{k}(x)\right\} \\
& \text { subject to } x \in S
\end{aligned}
$$

Here, $f_{1}(x), \ldots, f_{k}(x)$ are objective functions, $x=\left(x_{1}, \ldots, x_{n}\right)$ is the vector of decision variables and $S$ is the set of feasible solutions. Every point $x \in S$ is mapped to the point $\left(f_{1}(x), f_{2}(x), \ldots\right.$, $\left.f_{k}(x)\right)$ in $k$-dimensional objective space. Therefore, one can observe the objective set:

$$
F=\left\{\left(\left(f_{1}(x), f_{2}(x), \ldots, f_{k}(x) \mid x \in S\right)\right.\right.
$$

The notation „max” determines a simultaneous maximization of all the objective functions. If any objective function has to be minimized, the minimization of function $f_{i}(x)$ is performed by maximization of function $-f_{i}(x)$. According to the structure of feasible set $S$, discrete multi-criteria optimization problems do exist. In this PGTs problem, six decision variables exist, corresponding to the basic design parameters: $x=\left(x_{1}, x_{2}, x_{3}, x_{4}, x_{5}, x_{6}\right)=\left(z_{1}, z_{2}, z_{3}, n_{w}, m_{n}, b\right)$. Furthermore, there are four objective functions: volume $V(x)$, mass $m(x)$, efficiency $\eta(x)$ and production costs $T(x)$ :

$$
\begin{array}{ll}
f_{1}(x)=-V(x), & f_{2}(x)=-m(x), \\
f_{3}(x)=\eta_{p}(x), & f_{4}(x)=-T(x)
\end{array}
$$

Therefore, the mathematical model of nonlinear multi-criteria problem in concrete task, can be formulated as follows:

$$
\begin{aligned}
& \max \left\{f_{1}(x), f_{2}(x), f_{3}(x), f_{4}(x)\right\} \\
& \text { subject to } x \in S
\end{aligned}
$$

As multi-criteria optimization problems are mathematically ill-defined which can be seen from the definition, a criterion for selecting the optimal solution must be defined. The most important criterion for selecting these ,equally good" solutions is the Pareto optimality concept: The solution $x \in S$ is Pareto optimal if no solution $y \in S$ exists which maintains $f_{i}(x) \leq f_{i}(y)$ for all $i=1, \ldots, n$ and maintains strict inequality, i.e. $f_{i}(x)<f_{i}(y)$ for at least one index $i$. Determination of the Pareto optimal solutions set is the first stage in optimal solution finding. The optimal solution is selected in the next stage, where the weighted coefficients method is applied to select the optimal solution from the Pareto solutions set. 


\subsection{Weighted coefficients method}

For this method, the following scalarized problem must be set up:

$$
\begin{aligned}
& \max f^{M}(x)=w_{1} \cdot f_{1}^{0}(x)+\ldots+w_{m} \cdot f_{m}^{0}(x) \\
& \text { s.t. } \quad x \in S
\end{aligned}
$$

Weighted coefficients (or weights) $w_{i}$ are positive real numbers and $f_{i}^{0}(x)=\left(f_{i}^{0}\right)^{-1} f_{i}(x)$ are normalized objective functions where $f_{i}^{0}$ are normalizing coefficients [12]. All solutions obtained by using this method are Pareto optimal [12]. This model may be used regardless of the existence of priority functions or not [15].

The complete optimization procedure is implemented in the PLANGEARS software.

\section{Two-SPEED Two-CARrIER Planetary GEAR Trains}

\subsection{Two-carrier planetary gear trains structures and labeling method}

In cases where two-speed transmissions are required, a mechanism obtained by connecting two basic PGTs shown in Fig. 1 is one of the best suited design solutions. By joining two shafts of one PGT with two shafts of another PGT a mechanism is formed with four external shafts in total, Fig. 2.

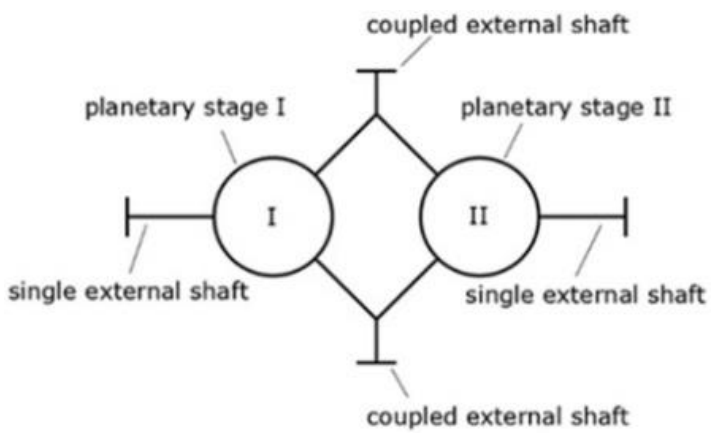

Fig. 2 Symbolic representation of a compound planetary gear train with four external shafts

The two component trains can be joined in in 12 different ways, resulting in a PGT with four external shafts [18]. An alphanumerical label (S11...S56) is attached to each of the 12 structural schemes, indicating the ways of connection between the shafts of the main elements of both component trains (Fig. 3). In every presented scheme it is also possible to place the brakes as well as the driving and the driven machine on external shafts in 12 different ways (V1...V12), corresponding to layout variants (Fig. 4).

The compound trains in consideration can be classified into three different groups according to whether the brakes are placed on the coupled shafts, on the single shafts or both on the coupled and the single shaft. 

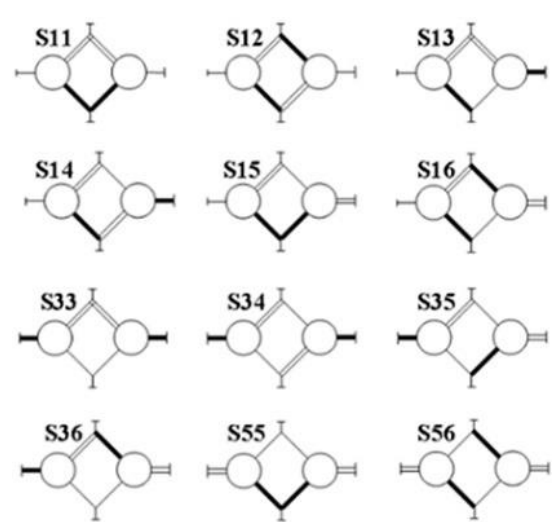

Fig. 3 Systematization of all schemes of two-carrier planetary gear train with four external shafts

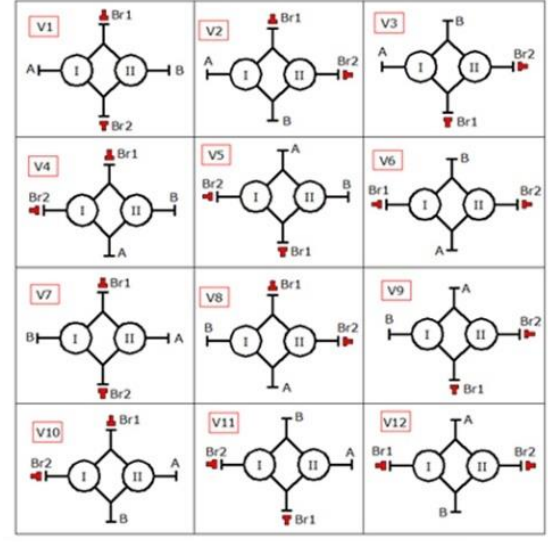

Fig. 4 Systematization of all layout variants (A-input shaft, B-output shaft)

\subsection{Operations of planetary gear trains with different layout variants}

By placing the brakes on two shafts, a braking system is obtained in which the alternating activation of the brakes shifts the power flow through the PGT, causing a change of the transmission ratio. Some PGTs of this type are described in $[5,7,18,19,20]$. The possible power flow paths for PGTs are analyzed, and functions of the transmission ratio for some trains of this type are deduced in $[5,18,19]$. 15 kinematic schemes of the considered type are presented in [6], and achievable values of transmission ratios and efficiencies are given. A computer program DVOBRZ for the selection of an optimal variant of similar multi-speed PGTs is described in $[5,18,19]$, and charts of shifting capabilities for all possible two-speed PGTs are given in [5].

Each variant has its own characteristics that determine the possibilities of transmission ratio changes. Some variants can be presumed to work in both transmission ratios as reducers and multipliers, while other variants work like a reducer with one ration and like a multiplier with the other ratio. Also, some variants change the direction of rotation when after a transmission ratio change, while other variants keep the direction of rotation after changing the transmission ratio. The transmission ratio of each PGT stage depends only on its basic transmission ratio (ideal torque ratio).

The compound train with brakes on the coupled shafts (layout variant V1 in the Fig. 4) with power flows when some of the brakes are active is symbolically shown in Fig. 5 by means of a Wolf-Arnaudov symbol. The green dotted line represents the power of relative motion. There are two possible directions: from the sun gear to the ring gear or from the ring gear to the sun gear. Also, the expressions for transmission ratios and efficiency obtained by using torque method when brake $\mathrm{Br} 1$ is activated are given on the left side, while the expressions for brake $\mathrm{Br} 2$ activated are given on the right side [5]. 


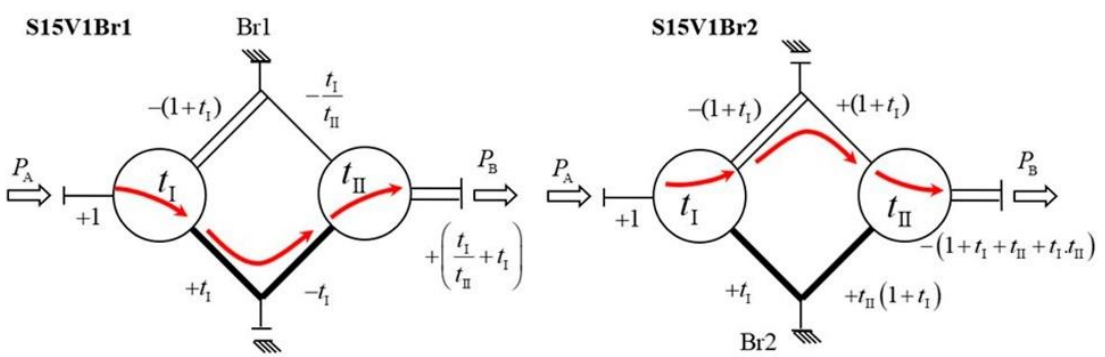

$i_{B r 1}=-\frac{T_{B}}{T_{A}}=-\frac{\frac{t_{I}}{t_{I I}}+t_{I}}{+1}=-\left(\frac{t_{I}}{t_{I I}}+t_{I}\right)=-\frac{t_{I}}{t_{I I}}\left(1+t_{I I}\right) ; i_{B r 2}=-\frac{T_{B}}{T_{A}}=-\frac{-\left(1+t_{I}+t_{I I}+t_{I} \cdot t_{I I}\right)}{+1}=+\left(1+t_{I}\right)\left(1+t_{I I}\right)$

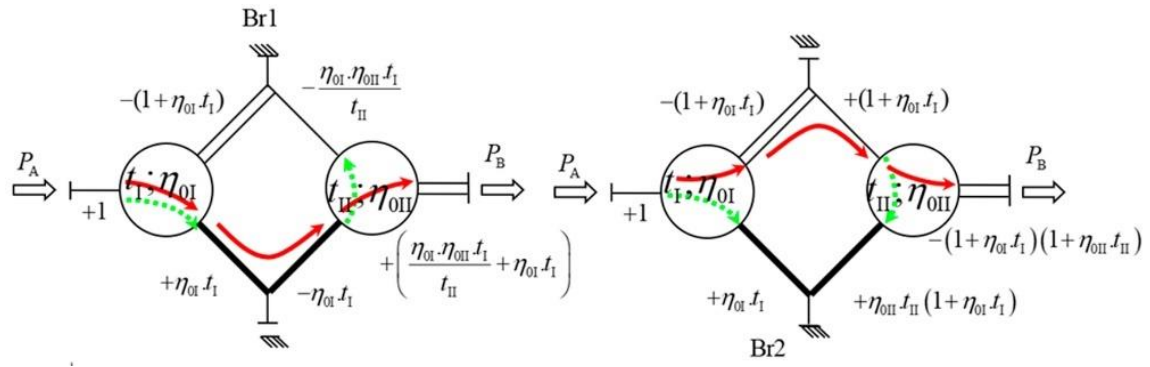

$$
\eta_{B r 1}=\frac{\left(T_{B}\right)_{\text {with loses }}}{\left(T_{B}\right)_{\text {without loses }}}=\frac{\frac{\eta_{0 I} \cdot \eta_{0 I I} \cdot t_{I}}{t_{I I}}\left(1+\frac{t_{I I}}{\eta_{0 I I}}\right)}{\frac{t_{I}}{t_{I I}}\left(1+t_{I I}\right)} ; \quad \eta_{B r 2}=\frac{\left(T_{B}\right)_{\text {with loseses }}}{\left(T_{B}\right)_{\text {without loses }}}=\frac{\left(1+\eta_{0 I} \cdot t_{I}\right)\left(1+\eta_{0 I I} \cdot t_{I I}\right)}{\left(1+t_{I}\right)\left(1+t_{I I}\right)}
$$

Fig. 5 Power flows on the Wolf-Arnaudov's symbol through the train with brakes on the coupled shafts

Regardless of which brake is applied, both the component trains operate actively, as seen in Fig. 5. The power input and output are on the single shafts. Also, the direction of the power flow is the same in both variants. When the upper brake $(\mathrm{Br} 1)$ is applied, the input element is the sun gear of the first stage. The power is transmitted through the ring gear of the first stage and the ring gear of the second stage to the output element - carrier of the second stage. In the other case, when the lower brake $(\mathrm{Br} 2)$ is applied, the path from the input element (sun gear of the first stage) to the output element (carrier of the second stage) includes the carrier of the first stage and the sun gear of the second stage.

As this transmission changes the direction of rotation with the transmission ratio, it is suitable for application in the machine tools which have a working motion with considerable load at low speed and a return motion to the initial position at high speed and light load. 


\section{RESULTS AND DiSCUSSION}

Variant S15V1(Figs. 6 and 7) was chosen to demonstrate the procedure of multi-criteria optimization application. A symbolic review of the transmission composition with kinematic scheme is shown in Fig. 6, and the path of the power flow through the transmission is shown in Fig. 7.

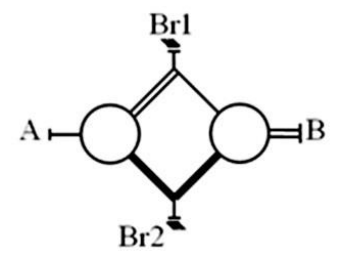

a)

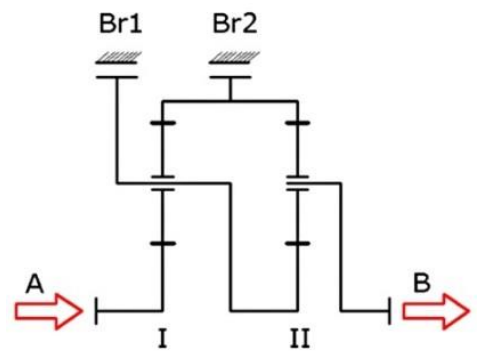

b)

Fig. 6 Symbolic review of transmission composition (a), kinematic scheme (b)

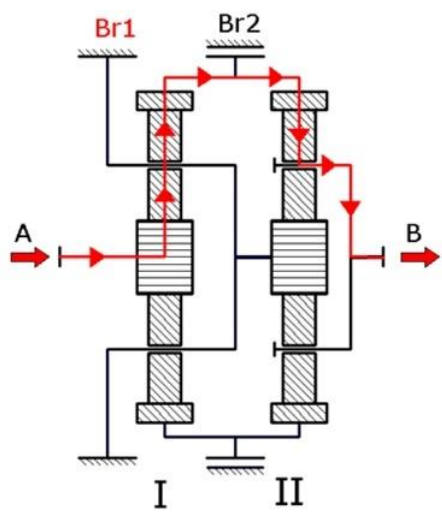

a)

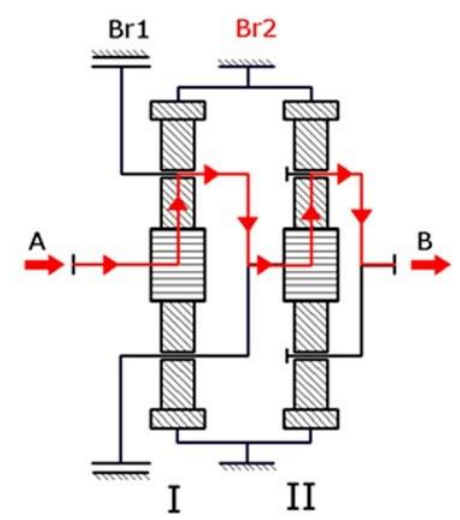

b)

Fig. 7 Power flow through the transmission on the conceptual scheme with brake $\mathrm{Br} 1$ applied (a) and with brake $\mathrm{Br} 2$ applied (b)

The type of transmission is selected according to the transmission requirements of the machine tool concerned. The necessary transmission ratios are: $i_{B r 1}=-6$ in one direction (with Br1activated) and $i_{B r 2}=40$ in the other (with $\mathrm{Br} 2$ activated). In this case, the ideal torque ratios for both the planetary gear stages can be defined from the shifting capabilities diagram, Fig. 8 [5]. 


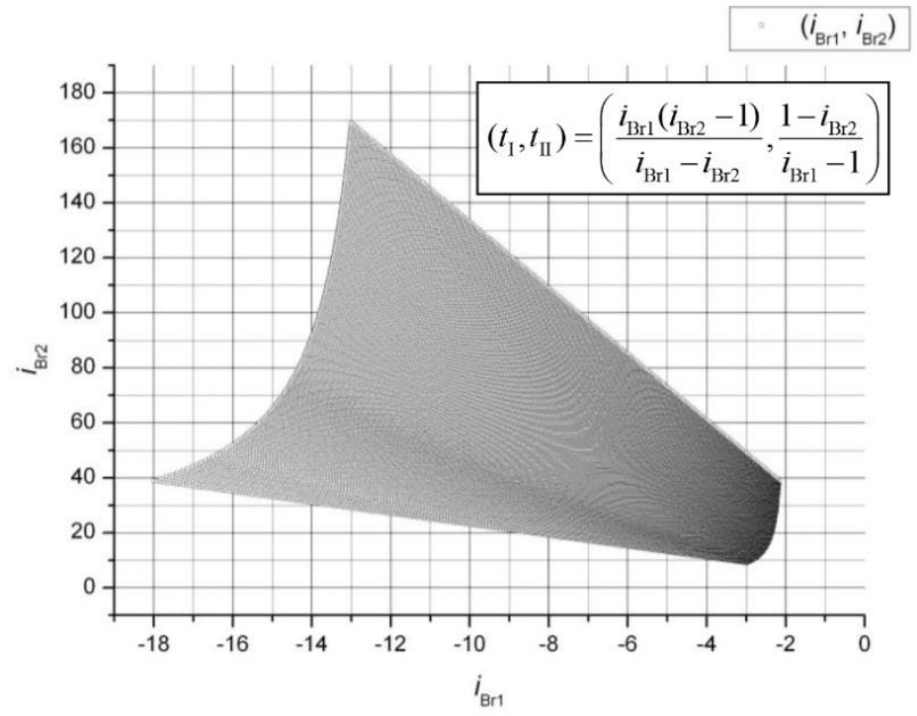

Fig. 8 Shifting capabilities diagram of compound trains S15V1

The transmission ratios are defined as functions of ideal torque ratios, $i_{B r 1}=-\left(t_{I} / t_{I I}\right)$. $\cdot\left(1+t_{I I}\right)$ and $i_{B r 1}=\left(1+t_{I}\right)\left(1+t_{I I}\right)$, so the ideal torque ratios are defined by using equations from Fig. 8: $t_{I}=5.09$ and $t_{I I}=5.57$ [5].

\subsection{The first compound gear train stage (I)}

With brake $\mathrm{Br} 1$ applied and brake $\mathrm{Br} 2$ turned off, the carrier of the first stage and sun gear of the second stage are immovable. The first stage is determined in this mode as the torque of the first stage is greater in this mode.

The input of the system (A) is the sun gear of the first stage (Figs. 6 and 7a). The output of the system (B) is the planet carrier of the second stage (Figs. 6 and 7). The power is transmitted to the ring gear of the first stage and then to the ring gear of the second stage and finally to the carrier of the second stage.

The input data required for the multi-criteria optimization application is: $i_{0}=-5.09$, $n_{\text {in }}=2850 \mathrm{~min}^{-1}, T_{\text {in }}=33.5 \mathrm{Nm}(P=10 \mathrm{~kW}), L=8000 \mathrm{~h}, K_{A}=1.25$, IT7 for all gears, material $z_{1} /$ material $z_{2} /$ material $z_{3}=20 \mathrm{MoCr} 4 / 20 \mathrm{MoCr} 4 / 34 \mathrm{CrNiMo6}, S_{H \min }=1.1, S_{F \min }=1.2, \Delta i=3 \%$, $z_{1}=15 \div 30$.

The feasible set consists of 834 solutions, from which 43 Pareto solutions are selected. By application of the weighted coefficient method with weighted coefficients: $w_{1}=0.5, w_{2}=0.0, w_{3}=0.0, w_{4}=0.5$ the solution shown in Table 1 is obtained, with a set of objective functions values shown in Table 2. The weighted coefficients are chosen in accordance with techno-economic optimization requirements. 
Table 1 Optimal solution of the first stage in the first case

\begin{tabular}{cccccc}
\hline \multicolumn{7}{c}{ Variable values } \\
\hline$x_{1}=z_{1}$ & $x_{2}=z_{2}$ & $x_{3}=z_{3}$ & $x_{4}=n_{w}$ & $x_{5}=m_{n}$ & $x_{6}=b$ \\
\hline 15 & 31 & -78 & 3 & 2 & 16 \\
\hline
\end{tabular}

Table 2 Objective functions for solution shown in Table 1

\begin{tabular}{cccc}
\hline$f_{1}$ in $\mathrm{mm}^{3}$ & $f_{2}$ in $\mathrm{kg}$ & $f_{3}$ & $f_{4}$ in $\min$ \\
\hline 305815.19 & 1.4529 & 0.9865 & 88.523 \\
\hline
\end{tabular}

By prioritizing other objective functions, i.e. by choosing other values for weighted coefficients, other solutions would become optimal.

The differences between solutions obtained in this way are logical. For example, the efficiency can be increased only with a large number of teeth considering Eq. (3) and the fact that efficiency is the only function that has to be maximized.

\subsection{The second compound gear train stage (II)}

With brake $\mathrm{Br} 2$ applied and brake $\mathrm{Br} 1$ turned off, the ring gears of both the stages are immovable. The second stage is determined in this mode as the torque at the sun gear of the second stage will be greater in this mode.

As in the previous mode, the input of the system (A) is the sun gear of the first stage and the output of the system (B) is the carrier of the second stage (Figs. 6 and 7b). The power is transmitted to the carrier of the first stage and then to the sun gear of the second stage and finally to the carrier of the second stage.

Since the ideal torque ratio of this stage is $t_{I I}=5.57$, the basic transmission ratio is $i_{0}=-t=-5.57$ and the transmission ratio of the second stage in this mode is $i=1-i_{0}=6.57$.

The input parameters of the second stage are equal to the output parameters of the first stage. Because of that, with brake $\mathrm{Br} 2$ applied, the ring gear of the first stage is also immovable, and the corresponding input number of revolutions at the second stage is calculated by means of the already defined first stage:

$$
n_{\text {inII }}=n_{\text {outI }}=\frac{n}{i}=\frac{2850}{6.2}=459.677 \mathrm{~min}^{-1}
$$

The torque on the sun gear of the second stage is calculated using the following equation:

$$
T_{1 I I}=T_{A}\left(1+t_{I}\right)=33.5 \cdot(1+5.2)=207.7 \mathrm{Nm}
$$

The other input data required for the multi-criteria optimization application in stage is equal to the one in the first stage: $L=800 \mathrm{~h}, K_{A}=1.25$, IT7 for all gears, material $z_{1} /$ material $z_{2} /$ material $z_{3}=20 \mathrm{MoCr} 4 / 20 \mathrm{MoCr} 4 / 34 \mathrm{CrNiMo6}, \quad S_{H \min }=1.1, \quad S_{F \min }=1.2$, $\Delta i=3 \%, z_{1}=15 \div 30$.

The feasible set consists of 1778 solutions, from which 43 Pareto solutions have been isolated.

By application of the weighted coefficient method with weighted coefficients: $w_{1}=0.5, w_{2}=0.0, w_{3}=0.0, w_{4}=0.5$ the solution shown in Table 3 was obtained, using a set of objective function values shown in Table 4. 
Table 3 Optimal solution of the second stage

\begin{tabular}{cccccc}
\hline \multicolumn{7}{c}{ Variable values } \\
\hline$x_{1}=z_{1}$ & $x_{2}=z_{2}$ & $x_{3}=z_{3}$ & $x_{4}=n_{w}$ & $x_{5}=m_{n}$ & $x_{6}=b$ \\
\hline 15 & 32 & -81 & 3 & 2.75 & 27 \\
\hline
\end{tabular}

Table 4 Objective function for solution shown in Table 3

\begin{tabular}{cccc}
\hline$f_{1}$ in $\mathrm{mm}^{3}$ & $f_{2}$ in $\mathrm{kg}$ & $f_{3}$ & $f_{4}$ in $\min$ \\
\hline 1040497.69 & 4.985 & 0.9865 & 121.85 \\
\hline
\end{tabular}

By comparing the design parameters of both the stages it can be concluded that the design parameters enable a compact transmission design that is very important for installation in a machine tool.

Also, the Pareto optimality concept as the criterion for selecting an equally good solution can be applied to compound PGT according to these criteria. Furthermore, the weighted coefficient method can be used for selecting the optimal solution from a Pareto set, and it can be adjusted to varying impacts of individual criteria functions.

Since the numbers of teeth of all gear are known, it is now possible to determine the realized transmission ratios and efficiency with brake $\mathrm{Br} 1$ applied and with brake $\mathrm{Br} 2$ applied, according to equations given in Fig. 5.

- Ideal torque ratio in the first stage:

$$
t_{I}=\frac{\left|z_{3}\right|}{z_{1}}=\frac{|-78|}{15}=5.2
$$

- Ideal torque ratio in the second stage:

$$
t_{I I}=\frac{\left|z_{3}\right|}{z_{1}}=\frac{|-81|}{15}=5.4
$$

- Transmission ratio with Br1 activated:

$$
i_{B r 1}=-\frac{t_{I}}{t_{I I}} \cdot\left(1+t_{I I}\right)=-\frac{5.2}{5.4} \cdot(1+5.4)=-6.16
$$

- Transmission ratio with $\mathrm{Br} 2$ activated:

$$
i_{B r 2}=+\left(1+t_{I}\right) \cdot\left(1+t_{I I}\right)=+(1+5.2) \cdot(1+5.4)=39.68 .
$$

The deviations of the actual and required transmission ratios are in the permissible range.

- Basic efficiency of the first stage:

$$
\eta_{0 I}=1-\frac{z_{3}}{z_{3}-z_{1}}\left(\frac{0.15}{z_{1}}+\frac{0.35}{z_{2}}+\frac{0.20}{z_{3}}\right)=1-\frac{(-78)}{(-78)-15}\left(\frac{0.15}{15}+\frac{0.35}{31}+\frac{0.20}{(-78)}\right)=0.984 \text {. }
$$

- Basic efficiency of the second stage:

$\eta_{0 I I}=1-\frac{z_{3}}{z_{3}-z_{1}}\left(\frac{0.15}{z_{1}}+\frac{0.35}{z_{2}}+\frac{0.20}{z_{3}}\right)=1-\frac{(-81)}{(-81)-15}\left(\frac{0.15}{15}+\frac{0.35}{32}+\frac{0.20}{(-81)}\right)=0.98442$. 
- Total efficiency with Br1 applied:

$$
\eta_{B r 1}=\frac{\frac{\eta_{0 I} \eta_{0 I I} t_{I}}{t_{I I}}\left(1+\frac{t_{I I}}{\eta_{0 I I}}\right)}{\frac{t_{I}}{t_{I I}}\left(1+t_{I I}\right)}=0.9816 .
$$

- Total efficiency with Br2 applied:

$$
\eta_{B r 2}=\frac{\left(1+\eta_{0 I} t_{I}\right)\left(1+\eta_{0 I I} t_{I I}\right)}{\left(1+t_{I}\right)\left(1+t_{I I}\right)}=0.97361
$$

It can be noticed that both efficiencies are very high $(97 \ldots 98 \%)$, and that the efficiency with brake $\mathrm{Br} 1$ on is slightly higher than the efficiency with brake $\mathrm{Br} 2$ on.

The second step can be performed by including the efficiency determined by defined gear tooth numbers. The calculation of input torque is shown here:

$$
T_{1 I I}=T_{A} \cdot\left(1+\eta_{0 I} \cdot t_{I}\right)=33.5 \cdot(1+0.984 \cdot 5.2)=204.91 \mathrm{Nm} .
$$

By applying the procedure of the optimal solution where only the value of the input torque has been changed, the solution shown in Table 5 is obtained using the objective function shown in Table 6. The feasible set consists of 1767 solutions, from which 44 Pareto solutions can be deducted. The weighted coefficients have the following values: $w_{1}=0.5, w_{2}=0.0, w_{3}=0.0, w_{4}=0.5$.

Table 5 Optimal solution of the second stage in the second step

\begin{tabular}{cccccc}
\hline \multicolumn{7}{c}{ Variable values } \\
\hline$x_{1}=z_{1}$ & $x_{2}=z_{2}$ & $x_{3}=z_{3}$ & $x_{4}=n_{w}$ & $x_{5}=m_{n}$ & $x_{6}=b$ \\
\hline 15 & 32 & -81 & 3 & 2.75 & 26 \\
\hline
\end{tabular}

Table 6 Objective function for solution shown in Table 5

\begin{tabular}{crlc}
\hline$f_{1}$ in $\mathrm{mm}^{3}$ & $f_{2}$ in $\mathrm{kg}$ & $f_{3}$ & $f_{4}$ in $\min$ \\
\hline 1001960.7375 & 4.80 & 0.984 & 119.611 \\
\hline
\end{tabular}

A difference is noticed in one variable only - face width. As the input torque is slightly lower, the expected face width will be smaller too. The distinction is negligible; therefore, it is not necessary to carry out the procedure including the efficiency determined by a defined number of teeth of the first stage.

\section{CONCLUSION}

An original method that combines two computer programs (DVOBRZ and PLANGEARS) for multi-criteria optimization of two-carrier two-speed PGTs with brakes on coupled shafts has been presented in this paper. These compound gear trains consist of two basic type of PGTs and have considerable application in systems which need different transmission ratios and direction changes (e.g. as machine tool gearboxes which work with 
a considerably greater transmission ratio in one direction and direction changing with a smaller transmission ratio in the other). The same procedure was successfully implemented in the optimal solution choice of two-carrier two-speed PGTs with brakes on single shafts, leading to a universal method of compound PGT optimization.

The optimal solution is determined by considering design parameters, such as mass and production cost as objective functions, and by using multi-criteria optimization and the weight coefficient method for choosing the optimal solution from the Pareto optimal solution set.

This approach can be successfully used for the basic PGT type and compound gear trains assembled from basic types, as shown in this paper. The results obtained using this procedure are in accordance with the literature on technical system optimization and indicate a good choice of optimization methods. Furthermore, this approach indicates a possibility for application to other PGT types.

\section{REFERENCES}

1. Tudose, L., Buiga, O., Jucan, D., Stefanache, C., 2008, Multi-objective optimization in helical gears design, The Fifth International Symposium about Design in Mechanical Engineering-KOD 2008, Novi Sad, Serbia, pp. 77-84.

2. Tkachev, A., Goldfarb, V., 2009, The concept of optimal design for spur and helical gears, The

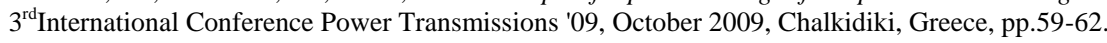

3. Rosić, B., 2001, Multicriterion optimization of multistage gear train transmission, Facta UniversitatisSeries Mechanical Engineering, 1(8), 2001, pp. 1107-1115.

4. Brüser, P., Grüschow, G., 1989, Otimierung von Planetengetrieben,Antiebstechnik, 2, pp. 64-67.

5. Troha, S., 2011, Analysis of a planetary change gear train's variants, (in Croatian), PhD Thesis, University of Rijeka, Engineering Faculty, Rijeka, Croatia.

6. Kudrjavtsev, V. N., Kirdyiashev, I. N., 1977, Planetary gears, (in Russian), Handbook, Mashinostroenie, Leningrad.

7. Arnaudow, K., Karaivanov, D., 2005, Systematik, Eigenschaften und Möglichkeiten von zusammengesetzten Mehrsteg-Planetengetrieben, Antriebstechnik, 5, pp. 58-65.

8. Ivanov, A.N., 1990, Evaluation of diametric dimensions of planetary gearboxes in the design phase, (in Russian),Vestnikmashinostroenie, 7, pp. 16-19.

9. Lechner, G., Naunheimer, H., 1999, Automotive Transmissions, Springer-Verlag, Heidelberg.

10. Jelaska, D., 2012, Gears and Gear Drives, University of Split, Croatia.

11. Stefanović-Marinović, J., Troha, S., Milovančević, M., 2017, An application of multicriteria optimization to the two-carrier two-speed planetary gear trains, Facta Universitatis-Series Mechanical Engineering, 15(1), pp. 85-95.

12. Stefanović-Marinović, J., Petković, M., Stanimirović, I., Milovančević, M., 2011, A model of planetary gear multicriteria optimization, Transactions of FAMENA, 35(4), pp. 21-34.

13. Sriatih, A., Yedukondalu, G., Jagadeesh, A., 2011, Mechanical efficiency of planetary gear trains: An Estimate, Mechanical Engineering Research, 1(1), pp. 97-102.

14. Del Castillo, J.M., 2002, Theanalytical expression of the efficiency of planetary gear trains, Mechanism and Machine Theory, 37, pp. 197-214.

15. Stefanović-Marinović, J., 2008, Multicriterionoptimization of planetary power transmission gear pairs, (in Serbian), PhD Thesis, University of Niš, Faculty of Mechanical Engineering, Niš, Serbia.

16. Niemann, G., Winter, H., 1989, Maschinenelemente, Band II, zweite völlig neubearbeite Auflage, Springer-Verlag Berlin.

17. International Organization for Standardization, 2006, Calculation of load capacity of spur and helical gears, International standard ISO 6336-2

18. Troha, S., Žigulić, R., Karaivanov, D., 2014, Kinematic operating modes of two-speed two-carrier planetary gear trains with four external shafts, Transactions of FAMENA, 38(1), pp. 63-76.

19. Troha, S., Lovrin, N., Milovančević, M., 2012, Selection of the two-carrier shifting planetary gear train controlled by clutches and brakes, Transactions of FAMENA, 36(3), pp. 1-12.

20. Troha, S., Petrov, P., Karaivanov, D., 2009, Regarding the optimization of coupled two carrier planetary gears with two coupled and four external shafts, Machine building and electrical engineering, 1, pp. 49-56. 